III.

\title{
Rupture of the Vaginal Wall with Protrusion of Small Intestine in a Woman 63 years of age ; Replace- ment, Suture, Recovery.*
}

\author{
By Andrew N. McGregor, M.D., F.F.P.S.G., \\ Assistant Surgeon to the Royal Infirmary, Glasgow.
}

Mrs. B., aged 63, was admitted into the Glasgow Royal Infirmary on the 23rd of October, 1906, suffering from a protrusion of intestine through the vulva, of three days' duration.

The patient gave the following history of the accident. On the 18th of October, she was carrying half a ton of coals into the house, when she "heard something give way." On the 19th, she felt out of sorts and took some whisky. That night, in trying to get out of bed, she fell across the back of a chair, astride, and sustained a severe blow on the perineum. She then observed a small quantity of blood coming from the vagina, and, on rising the following morning, became aware of a mass protruding from the vaginal orifice. She tried to press the mass back, but failed. On Monday the 22nd, she called in a doctor-Dr. Kirkland of Airdrie-who advised her removal to hospital.

On admission, her temperature was $98^{\circ} \mathrm{F}$.; the pulse rate was 96 per minute, and of moderate tension. A mass of bowel was seen protruding from the vagina. It was covered with a fibrinous exudate and the coils were adherent. A whitish, foul-smelling discharge lay in the sulci between the folds of intestine and in the vagina. There were no visible wounds or bruises of the thighs, perineum, or vulva. A slight amount of tenderness and some distension of the lower part of the abdomen were observed. There were no signs of intestinal obstruction.

The patient had borne 10 children, but had had no prolapse or trouble of any kind with her uterus or vagina.

She was anæsthetized by chloroform, and a more thorough examination of the parts then revealed the presence of a tear in the vagina. The long axis of the wound lay transversely across the posterior wall of the vagina immediately behind the cervix, and was more extensive on the right side than on the left. The adhesions between the folds of bowel were separated, and the bowel was care-

* Communicated to the Glasgow Pathological and Clinical Society, January 14th, 1907. 
fully washed in saline solution before being replaced into the pouch of Douglas. The vagina was also carefully cleansed and the wound in its posterior wall sutured, from left to right, for about two-thirds of its extent. The remainder of the opening was packed with iodoform gauze, and the vagina was filled with a similar material. The extent of the extruded bowel was about 18 inches, and its bulk was so great before operation that urine must have passed over it during micturition. The edges of the wound presented a torn appearance.

On the day after the operation it was noted that the temperature had risen to $101 \cdot 2^{\circ} \mathrm{F}$. and the pulse to 108 . The whole abdomen was somewhat swollen and tender. The vaginal packing was removed and found saturated with pus. Fresh packing was introduced. The urine was drawn off by catheter.

The vaginal dressing was again changed on the third day, and thereafter, the dressing was removed daily until the twelfth day. The plug of gauze was removed from the wound on the seventh day and a douche of hot boric solution was administered daily at each change of the vaginal packing. The bowels acted in response to enemata 48 hours after the operation, and during the ensuing two days there was some diarrhœa. The temperature averaged $998^{\circ} \mathrm{F}$. during the first 12 days and then declined to normal in two days after the discontinuance of the vaginal packing. On the 16th day it was noted that the wound was entirely healed and that there was no vaginal tenderness except over the scar. There was still a slight leucorrhcea. The patient was allowed up on the 18th day and left the hospital three days later.

Although rupture of the vagina with protrusion of the intestines as an accident of childbirth has been followed by recovery in a number of cases, there are points of special interest in this case, notably those of causation and treatment.

In the first place the mechanism of the injury is somewhat obscure. Rupture of the vagina in the great majority of cases occurs during pregnancy and parturition, and the reported cases may be classified into the following groups according to their causation:-

1. During pregnancy and parturition.

(a) The "spontaneous" variety.

(b) Direct injury by the advancing fœtus.

(c) Laceration by instruments or manipulation. 
2. Apart from pregnancy.

(a) During coïtus

(b) Direct injury by instruments and foreign bodies.

(c) During replacement of malpositions of the uterus.

(d) By the upward growth of fibroid or ovarian tumours.

(e) By indirect violence (?).

The cases included in class 1 -during pregnancy and parturitionthough occurring under conditions which do not directly apply to this case, are worthy of consideration in so far as their causes are concerned. In particular that variety called " spontaneous" rupture of the vagina has led to the enunciation of theories which are interesting and which may contribute something towards the elucidation of the mechanism of indirect ruptures of the vagina.

The word "spontaneous" was applied to ruptures occurring during pregnancy, to indicate the absence of direct injury such as might occur during parturition. Amongst the earlier writers on the subject is Dr. R. Doherty. He relates the case of a woman of 36 years who had a fatal rupture of the vagina during the last months of pregnancy before labour had begun (Dublin Journ. of Med. Science, 1845, vol. xxvii., pp. 325-356). The tear corresponded to the right iliopectineal line, which, however, was not unusually sharp. The peritoneum was not torn but death resulted from hæmorrhage. There had been seven previous pregnancies; the pelvis was contracted; the fourth labour had been complicated by a funis and foot presentation, delivery being effected by perforation of the head and the use of the crotchet. Dr. Doherty believed that the predisposing cause was an unhealthy state of the vagina as the result of that difficult labour, and that the direct cause was the turning of the patient in bed, inducing a change of position of the uterus.

Wiltshire reports a case of spontaneous rupture of the vagina (Trans. Obstet. Society, London, 1876, vol. xvii., p. 362) in a woman of 38 , who had previously borne nine children. When seen after labour had been in progress 18 hours the right arm and leg presented, and there was a laceration of the vagina, large enough to admit three fingers, just behind and to the right of the cervix. The promontory of the sacrum was found to jut out into the brim of the pelvis. There was little hæmorrhage; the bowel could be felt through the tear, but no blood-clots. The patient recovered without direct treatment of the tear. Wiltshire gives the cause as threefold, viz., $(a)$ the undue projection of the sacrum, $(b)$ the malposition of the fœtus, $(c)$ and the violent efforts of the patient to effect delivery. Wiltshire quotes 
Ingleby (Obstetric Med., p. 177) to the effect that a want of correspondence between the uterine and vaginal axes is a cause of laceration during parturition. M'Clintock says (Dublin Quarterly Journal of Med. Science, May 1866): "It is worthy of notice that in nearly all the cases of spontaneous laceration the direction of the tear was more circular than longitudinal," and Wiltshire definitely states that circular tears occurring just behind the cervix are due to "spontaneous" rupture.

Cases are on record, notably that reported by Henry (Lancet, 1857, vol. ii., p. 325), in which the laceration of the vagina was so great that the foetus was found in the peritoneal cavity. The child was delivered along with the placenta, membranes, and some large clots, and the mother recovered in a few weeks after some peritonitis. In contrast to these complete ruptures into peritoneal cavity there are cases noted in which the rupture was extraperitoneal, and it is probable that many small lacerations escape notice.

The question naturally arises as to whether this patient, during her ten pregnancies and deliveries, had experienced a partial rupture of the vagina, the scar of which gave way at the time of the accident. The patient, however, does not admit that any difficulties were experienced in her labours, and there is no pelvic deformity, such as those noted above, which would interfere with the normal course of labour. For similar reasons we are able to exclude partial rupture from the other causes such as laceration by the fœtus or by instruments, and there is no evidence of inflammatory or malignant disease which would diminish the strength of the vaginal wall.

Considering the causes of rupture of the vagina apart from pregnancy, the possibility of coitus or criminal assault was suggested. The patient denied the possibility of either of these, and no evidence of such causes was obtained by Dr. Kirkland, who first saw her after the accident, and who, subsequently, after her return home, investigated this matter. Direct injury by a foreign body must also be excluded unless one admits the possibility of a part of the chair, across which she fell, entering the vagina and causing a tear at its upper and posterior limit without bruising or tearing the perineum, vulva, or other part of the vagina than that torn.

Some cases are recorded in which rupture of the vagina was due to replacement of a displaced uterus. That recorded by Fehling is perhaps the most striking (Archiv für Gynäk., Berlin, 1873-4, vol. vi., p.103, and Brit. and Foreign Med. Chir. Review, 1872-74, p. 513). He was called to a woman, æt. 63 , who had suffered from prolapse for 30 years. Some hours previous to his visit, as she was carrying a pail of water 
up some steps, the womb prolapsed. She tried to replace it and used some force. She felt something give way and the intestine protruded. Dr. Fehling found the intestine protruding through the vulvar orifice, the mass being as large as a man's head. The rent was found in the posterior vaginal wall, and an unsuccessful attempt made to replace the bowel. Puncture was tried, with no good result, and the patient died in 11 hours after the accident. Post mortem there was no sign of peritoneal inflammation. Dr. Fehling could find no previously recorded similar case. Dr. Gaillard Thomas had a case where the vagina was ruptured during an attempt to restore an inverted uterus (Schroeder, in von Ziemssen's Cyclopadia of Medicine, vol. x., pp. 220-1).

In the present instance the patient has had no prolapse of or interference with the uterus. Fehling's case shows that the vaginoperitoneal partition may be very easily ruptured since the force that a woman could employ in the act of replacing her uterus was sufficient to tear it.

Schroeder (loco cit.) states that the upward growth of ovarian or fibroid tumours may cause rupture of the cervix in the unimpregnated uterus, and he also says that Gotthardt met with a case of rupture of the vagina due to a fall. There is no tumour formation in the present case, but there is the history of a fall.

Referring to the story of the accident it is evident that there were two occurrences, for on the $18 \mathrm{th}$ of October she carried half a ton of coals into the house and "heard something give way," and on the following day she felt out of sorts and took some whisky, and later fell out of bed astride a chair and sustained a blow on the perineum. The sensation experienced during the work of carrying coals may have been due to the occurrence of a prolapse of the uterus, and the subsequent discomfort to that malposition. It is unlikely that the vagina had been ruptured else there would have been a history of hæmorrhage or of bowel protrusion. It was not till after the fall out of bed, if the story be correct, that the protrusion of bowel occurred. Theoretically that blow on the perineum should have accentuated the prolapse of the uterus, but, if the tear was due to that cause the uterus must have returned to its position for the bowel filled the vagina. The evidence in the case seems to indicate that the rupture was due to indirect violence and a review of the cases cited gives support to that view.

The second point of interest is that of treatment. The three courses which suggested themselves were:-(1) Excision of the pro- 
jecting mass and return of the stump through the wound. (2) Replacement of the prolapsed bowel. (4) Abdominal section.

The first alternative was rejected because of the difficulty of access to the non-contaminated bowel and the necessity for its being drawn into the vagina during the operation. This would have extended the area of infection. Then, too, a resection of bowel in a patient suffering from profound shock induced by a three days' exposure of bowel was hardly likely to have been successful.

It was felt that the second plan was the most feasible for the reason that the lapse of time had been sufficient to allow of the formation of adhesions, and of omental protection taking place, so as to shut off the pouch of Douglas from the general peritoneal cavity. There remained, however, the difficulty of cleansing as well as that of returning the bowel to the pouch of Douglas. The adherent loops of bowel were separated and washed with saline solution. The vagina was douched gently with a similar lotion, and thereafter a careful palpation revealed the position of the tear.

Gentle traction was made on the bowel at its point of emergence through the wound so that another inch or so escaped, and, as there was no evidence of strangulation or gangrene, the whole mass was systematically replaced. During its manipulation the bowel diminished in size considerably with the accompaniment of a gurgling sound.

The cervix was caught with a volsella and held downwards and forwards so as to facilitate the insertion of the sutures. This stage was accomplished without much difficulty. Complete closure of the wound was not attempted, a gap amounting to about a third of its extent being left for the insertion of a loose gauze drain. The uterus was released; the vagina was swabbed dry and filled with iodoform gaure.

The third course might have been necessary had it been found impossible to return the bowel without the aid of a hand in the abdomen. Theoretically it seems to offer a better chance of success than the method of puncture tried by Fehling (loco cit.) the disadvantage being the separation of adhesions and perhaps the removal of a protecting omental adhesion.

A review of the history of this patient and of the records of published cases of rupture of the vagina in which recovery took place without suture of the tear seem to show that the good results are due to the isolation of Douglas's pouch. This factor is therefore an important consideration in the choice of an operation where there is evidence of bowel contamination. 
In conclusion this case may be summarized as one in which a rupture of the vagina occurred in the position where the so-called "spontaneous" ruptures, which happen during pregnancy, are situated. The patient was a multiparous woman without any history of uterine or vaginal trouble. She was aged; the uterus was normal; and there were no tumours of that organ or its adnexa. The predisposing cause was possibly an undue thinness of the posterior vaginal wall. A prolapse of the uterus probably occurred during the heavy work of carrying the coal, and the fall during the night may have produced the rupture either owing to the undue mobility of the uterus or to the altered intra-abdominal tension and the weakened vaginal wall. The reposition of the protruded bowel was undertaken as the only obvious course, and the success of this proceeding was presumably due to the prior formation of protective adhesions, shutting off the pouch of Douglas and its contents from the general peritoneal cavity. It is remarkable that a woman of 63 years of age should have had sufficient vitality to withstand the shock of the protrusion of 18 inches of bowel through a tear in her vagina, should survive the shock of the replacement, and should escape without a general septic peritonitis.

Nearly three months after the accident, the patient was examined by Dr. J. K. Kelly, who reported to me as follows : - "I have just examined Mrs. B., and have to report that I find the condition of the genital organs normally senile. The only abnormality is a linear scar in the posterior vaginal fornix. There is no tumour in Douglas's pouch to indicate abnormal adhesions there. From the condition of the parts I consider that the cause of the rupture for which you operated must have been some direct violence and not a mere increase, however sudden, of the intra-abdominal pressure." 